\title{
Zygomycosis Presenting as Acute Myocardial Infarction during Hematological Malignancies
}

\author{
Satoru Joshita ${ }^{1,2}$, Kiyoshi Kitano ${ }^{1}$, Tadanobu Nagaya ${ }^{1,2}$, Atsushi Kamijo ${ }^{1}$, \\ Koh Nakazawa ${ }^{3}$ and Fumihiro Ishida ${ }^{4}$
}

\begin{abstract}
Here we report two patients with hematological malignancies associated with complications of fatal cardiac zygomycosis. The first case, a 72-year-old man with myelodysplastic syndrome being treated with low-dose cytarabine, died of sudden cardiac arrest. An autopsy revealed disseminated zygomycosis accompanied with occlusion of the coronary artery by fungal thrombi. The second case, a 52-year-old woman with acute lymphoblastic leukemia, developed febrile neutropenia and skin eruptions with induration on the face and extremities during the first induction chemotherapy. She experienced sudden bradycardia with unstable hemodynamics and died of acute myocardial infarction. Histological examination of a skin biopsy demonstrated zygomycosis. In light of the above, it should be kept in mind that cardiac zygomycosis might occur in hematologically compromised patients presenting with acute myocardial infarction.
\end{abstract}

Key words: zygomycosis, acute lymphoblastic leukemia, myelodysplastic syndrome, myocardial infarction

(Inter Med 47: 839-842, 2008)

(DOI: 10.2169/internalmedicine.47.0832)

\section{Introduction}

Zygomycosis is an invasive, opportunistic infection caused by the fungal order Mucorales or Entomophtorales. It belongs to the Zygomycetes class of true fungi and is characterized by aseptate or nearly aseptate hyphae (1). Zygomycosis is rare, but occurs with increased frequency during chemotherapy-induced prolonged neutropenia and solid organ or bone marrow transplantation. The mortality of zygomycosis remains high (2). Infections due to Mucormycetes include rhinocerebral, pulmonary, cutaneous, gastrointestinal, and disseminated diseases $(3,4)$. Zygomycosis has a clear affinity for blood vessels, often causing thrombosis and infarction, but cardiac zygomycosis is a rare complication (57). However, we present two patients with zygomycosis complications who died of acute myocardial infarction in association with hematological malignancies, highlighting the need to remember the possibility of zygomycosis in immuno-compromised patients.

\section{Case Report}

\section{Case 1}

A 72-year-old man was admitted to our hospital suffering from fever and cough. On examination, his temperature was $37.3^{\circ} \mathrm{C}$. Coarse crackles were heard on auscultation on the right side of his chest. Laboratory tests showed a white

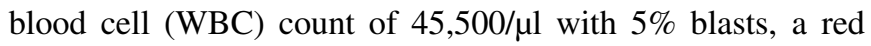
blood cell count of $242 \times 10^{4} / \mu$, and a platelet count of $3.3 \times$ $10^{4} / \mu \mathrm{l}$. His hemoglobin value was $7.7 \mathrm{~g} / \mathrm{dl}$. Renal dysfunction was also detected as a serum level of creatinine at 1.6 $\mathrm{mg} / \mathrm{dl}$. He was diagnosed as having myelodysplastic syndrome (refractory anemia with excess blasts) following a bone marrow examination. Chromosomal analysis showed normal karyotype. Imipenem cilastatin at $1 \mathrm{~g}$ and fluconazole at $100 \mathrm{mg}$ daily were administered, but cough and fever continued intermittently. His WBC count gradually increased to $126,000 / \mu \mathrm{l}$ with $2 \%$ blasts before $10 \mathrm{mg} / \mathrm{m}^{2}$ cyta-

\footnotetext{
${ }^{1}$ Department of Internal Medicine, Matsumoto National Hospital, Matsumoto, ${ }^{2}$ Department of Internal Medicine, Division of Gastroenterology and Hepatology, Shinshu University School of Medicine, Matsumoto, ${ }^{3}$ Department of Laboratory Medicine, Matsumoto National Hospital, Matsumoto and ${ }^{4}$ Department of Internal Medicine, Division of Hematology, Shinshu University School of Medicine, Matsumoto Received for publication December 17, 2007; Accepted for publication January 29, 2008 Correspondence to Dr. Satoru Joshita, joshita@hsp.md.shinshu-u.ac.jp
} 


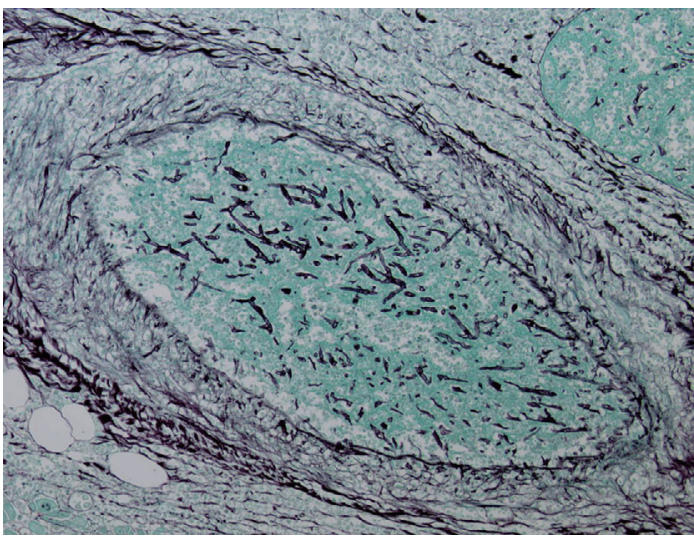

A

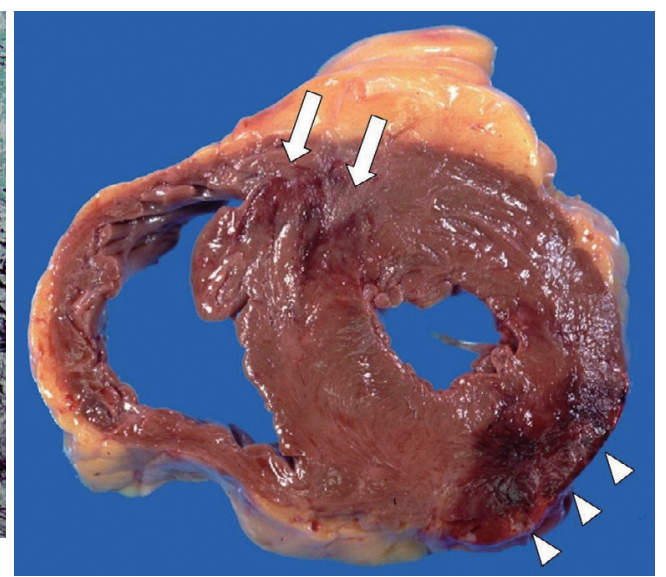

B

Figure 1. A: Histological finding shows occlusion of the coronary artery by fungal hyphae (Case 1, Grocott staining, $\times 10)$.

B: Macroscopic finding of the heart at autopsy shows anteroseptal (arrows) and lateral (arrowheads) myocardial infarction (Case 1).

rabine (Ara-C) therapy was started. Daily doses of $100 \mathrm{mg}$ cytarabine ocfosfate were taken orally as maintenance treatment afterwards. Four months after admission, bloody pleural effusions appeared on his right side. Administration of amphotericin B (AmB) at a dose of $20 \mathrm{mg}$ per day was started because of raised serum $\beta$-D glucan values of 36.3 $\mathrm{pg} / \mathrm{ml}$ (normal range; $<20.0 \mathrm{pg} / \mathrm{ml}$ ). One month after, the patient complained of abdominal pain around the umbilicus and rib bone pain on his left side. A new coin lesion was detected in left middle lung field $X$ rays on day 205. His left anterior chest pain continued and echocardiography revealed hypokinesis at the cardial septal wall. In the early morning of day 208, low blood pressure, sinus bradycardia, and cyanosis all occurred suddenly. His hemodynamics continued to deteriorate despite being immediately put on a respirator and intravenously administered catecholamine, and he died four hours later. Blood cultures had been examined several times during the clinical course but were all negative for the Zygomycetes. The diagnosis following autopsy was disseminated zygomycosis with the findings as follows; occlusion of coronary artery by fungal hyphae (Fig. 1A), anteroseptal to lateral myocardial infarction (Fig. 1B), and systemic fungal thrombi with hyphae invasion in the cardiac ventricular wall, left lung, jejunum, ileum, colon, and kidney.

\section{Case 2}

A 52-year-old woman was admitted to our hospital suffering from general fatigue. Since her father had died two months earlier, she had been helping with her family's agricultural fieldwork and frequently played with her grandchildren in the sandbox.

On examination, the patient was $166 \mathrm{~cm}$ tall and weighed $50.5 \mathrm{~kg}$. Her body temperature was $37.4^{\circ} \mathrm{C}$. She showed hepatosplenomegaly, and lymphoadenopathy was also detected.

Laboratory tests showed a WBC count of $123,790 / \mu$ with $98 \%$ blasts, a red blood cell count of $233 \times 10^{4} / \mu 1$, and a platelet count of $1.8 \times 10^{4} / \mu \mathrm{l}$. Her hemoglobin value was 6.8 $\mathrm{g} / \mathrm{dl}$ and hematocrit was $21.8 \%$. Blood chemistry showed lactate dehydrogenase at 1,004 U/l. A bone marrow biopsy revealed marked hypercellularity with peroxidase-negative monotonous proliferating cells. Translocation (9; 22) (q34; q11) was noted in chromosomal analysis and the amount of minor-bcr / abl chimera messenger RNA was $8.5 \times 10^{4}$ copies/ $\mu$ gRNA. The patient was subsequently diagnosed as having $\mathrm{Ph}$-positive acute lymphoblastic leukemia and received the first induction chemotherapy using an isolator for prevention of fungal infection on day 3 of admission with cyclophosphamide, doxorubicin hydrochloride, vincristine sulfate, prednisolone, and imatinib mesilate (8). Itraconazole capsules at $200 \mathrm{mg}$ daily were used as prophylactics against mycotic infection from the time of admission. On day 21, she experienced continuous abdominal pain and became constipated, bearing a paralytic ileus on abdominal X-ray, and then displayed marked fever on day 23. Cefepime at $4 \mathrm{~g}$ daily and granulocyte-colony stimulating factor (G-CSF) were started. Blood cultures were tested several times after the onset of fever but were all negative for Zygomycetes. Purpuric skin lesions with induration (Fig. 2A) appeared on the face and extremities on day 25, which were biopsied and sent for tests. In the early morning of day 27 , bradycardia suddenly occurred and she fell into shock. Her hemodynamics continued to deteriorate despite being immediately put on a respirator and intravenously administered catecholamine. Electrocardiograms showed bradycardial escape rhythm and ST segment elevation in leads II, III, and aVF. Serological troponin $\mathrm{T}$ and heart-type fatty acid-binding protein were both positive. The patient suddenly entered ventricular tachycardia and ventricular fibrillation and passed away three hours later. An autopsy was not performed. Three days following her death, the histological findings of the skin biopsy showed fungal hyphae located in an arterial vessel with a fibrin thrombus and outside the vessel (Fig. 2B), leading to a diagnosis of zygomycosis. 

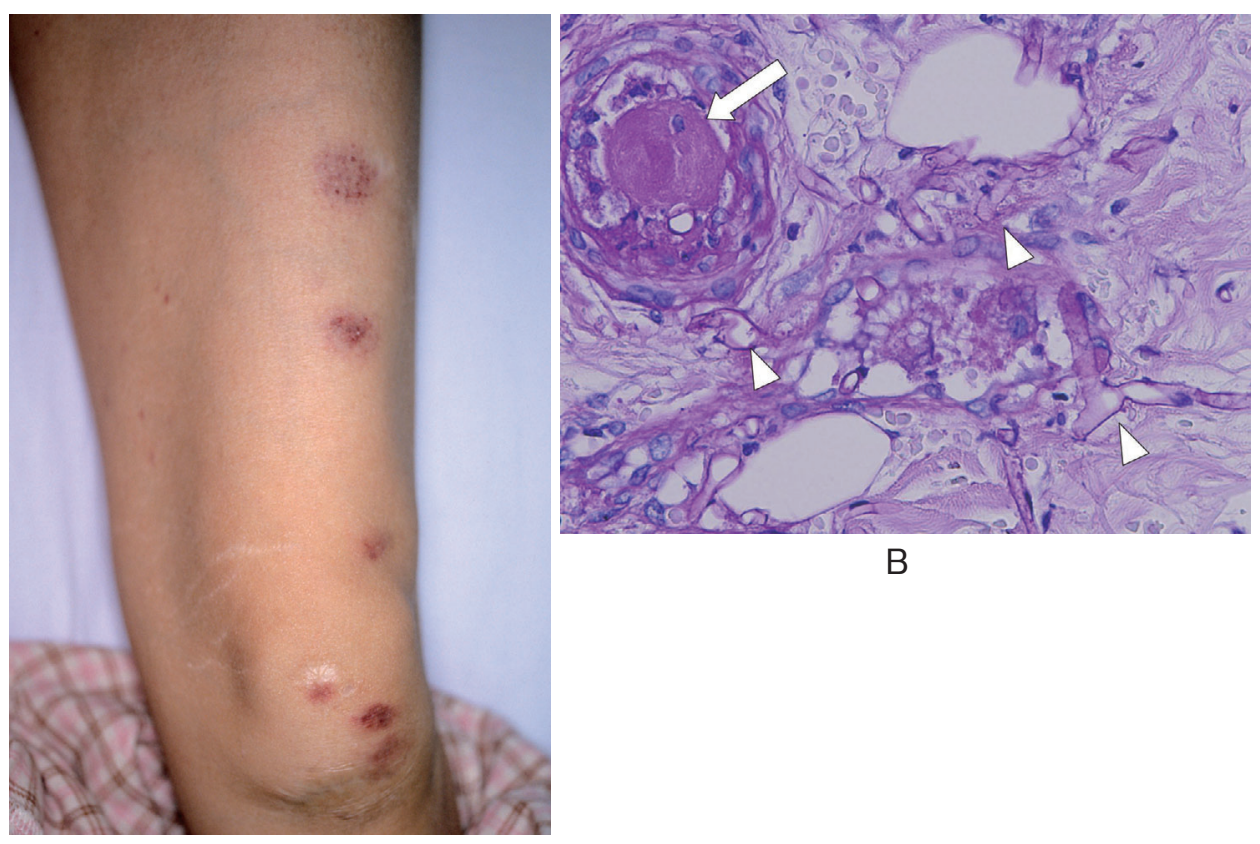

B

A

Figure 2. A: Purpuric skin lesions with induration, nodules, plaques, and echthyma appeared on the lower extremities (Case 2).

B: Skin biopsy shows fungal hyphae with wide, hyaline, ribbon-like, predominantly aseptate, and wide-angled branching, located both within an arterial vessel with a fibrin thrombus (arrow) and around it (arrowheads) (Case 2, periodic acid-Schiff staining, $\times 40$ ).

\section{Discussion}

Zygomycetes fungi are ubiquitous in nature and can be found on decaying vegetation and in the soil, meaning all humans are exposed to these fungi in their day-to-day activities. The most common route of transmission for Zygomycetes is inhalation of spores from the environment (9). Specifically in case 2 , the patient had contact with soil prior to admission, which might have constituted a route of infection. Therefore, it is essential to get pre-hospital history in detail, especially about the home environment, before initiation of chemotherapy for hematological malignancies.

The diagnosis of zygomycosis relies upon the identification of organisms in tissues by culture and/or histopathological examination, and not upon serological examinations, such as those for serum $\beta$-D glucan. In our cases, we identified Zygomycetes hyphae histologically either at autopsy or through a skin biopsy section. In case 1 , high serum $\beta$-D glucan was noticed and indicated a concurrent infection of Candida albicans confirmed by a sputum culture.

The risk of systemic fungal infection with Zygomycetes increases noticeably during a prolonged neutropenic phase $(9,10)$. Disseminated zygomycosis is a rare, usually fatal infectious complication in immunocompromised patients, and constitutes up to $40 \%$ of zygomycosis in patients with hematological malignancy (6). The most characteristic histological features of zygomycosis are perivascular and blood vessel invasions that result in arterial thrombosis and subsequent tissue necrosis. Cardiac involvement of zygomycosis is relatively rare; it has been documented that only one of 59 patients (6) and two of 34 patients (5) with zygomycosis had cardiac involvement as the primary site of infection. However, cardiac zygomycosis seems to be a component of disseminated zygomycosis (11). In another report, two cases of cardiac involvement of zygomycosis were documented to have disseminated zygomycosis as well (7), and all five cases of cardiac zygomycosis had involvement of lungs and other organs in yet another study (12). It has also been reported that the clinical manifestations of cardiac zygomycosis with predominant myocardial involvement are quite nonspecific (7). In the present study, case 1 suffered from chest pain, but no chest symptoms were noted in case 2 with the exception of a dull, continuous abdominal pain.

A critical component of treatment for zygomycosis is early surgical intervention accompanied with high-doses of $\mathrm{AmB}$ or liposomal AmB and improvement of general condition with immune reconstitution $(9,13,14)$. Several antifungal agents, such as voriconazole or caspofungin, which have been evaluated as empiric therapies for patients with fever and neutropenia (15-18), are not effective against Zygomycetes (19). It appears the patient in case 1 did not receive a sufficient dose of AmB due to renal dysfunction. The features of zygomycosis were likely overlooked in case 2 due to the side effects induced by chemotherapy, especially in the induction phase, which was complicated by febrile neutropenia. Gubarev et al described a 37-year-old male who underwent successful treatment of cardiac zygomycosis with high-dose liposomal AmB (20). Liposomal AmB has been shown to be as effective as conventional $\mathrm{AmB}$, but with less 
infusion-related toxicity, and nephrotoxicity (21).

In conclusion, cardiac zygomycosis is rare, but should be kept in mind when treating hematologically compromised hosts with complicating life-threatening cardiac diseases, such as AMI or irreversible arrhythmia.

\section{Acknowledgement}

We thank Trevor Ralph for his editorial assistance.

\section{References}

1. Ribes JA, Vanover-Sams CL, Baker DJ. Zygomycetes in human disease. Clin Microbiol Rev 13: 236-301, 2000.

2. Roden MM, Zaoutis TE, Buchanan WL, et al. Epidemiology and outcome of zygomycosis: A review of 929 reported cases. Clin Infect Dis 41: 634-653, 2005.

3. Sugar AM. Mucormycosis. Clin Infect Dis 14 Suppl 1: S126S129, 1992.

4. Kontoyiannis DP, Lionakis MS, Lewis RE, et al. Zygomycosis in a tertiary-care cancer center in the era of Aspergillus-active antifungal therapy: a case-control observational study of 27 recent cases. J Infect Dis 191: 1350-1360, 2005.

5. Pagano L, Ricci P, Tonso A, et al. Mucormycosis in patients with haematological malignancies: a retrospective clinical study of 37 cases. GIMEMA Infection Program (Gruppo Italiano Malattie Ematologiche Maligne dell'Adulto). Br J Haematol 99: 331-336, 1997.

6. Pagano L, Offidani M, Fianchi L, et al. GIMEMA (Gruppo Italiano Malattie EMatologiche dell'Adulto) Infection Program. Mucormycosis in hematologic patients. Haematologica 89: 207-214, 2004.

7. Jackman JD Jr, Simonsen RL. The clinical manifestations of cardiac mucormycosis. Chest 101: 1733-1736, 1992.

8. Yanada M, Takeuchi J, Sugiura I, et al. Japan Adult Leukemia Study Group. High complete remission rate and promising outcome by combination of imatinib and chemotherapy for newly diagnosed BCR-ABL-positive acute lymphoblastic leukemia: a phase II study by the Japan Adult Leukemia Study Group. J Clin Oncol 24: 460-466, 2006.

9. Brown J. Zygomycosis: an emerging fungal infection. Am J Health Syst Pharm 62: 2593-2596, 2005.

10. Gerson SL, Talbot GH, Hurwitz S, et al. Prolonged granulocytopenia: the major risk factor for invasive pulmonary aspergillosis in patients with acute leukemia. Ann Intern Med 100: 345-351, 1984.

11. Benbow EW, McMahon RF. Myocardial infarction caused by cardiac disease in disseminated zygomycosis. J Clin Pathol 40: 70-
74, 1987.

12. Virmani R, Connor DH, McAllister HA. Cardiac mucormycosis. A report of five patients and review of 14 previously reported cases. Am J Clin Pathol 78: 42-47, 1982.

13. Kontoyiannis DP, Wessel VC, Bodey GP, Rolston KVI. Zygomycosis in the 1990s in a tertiary-care cancer center. Clin Infect Dis 30: 851-856, 2000.

14. Gonzalez CE, Rinaldi MG, Sugar AM. Zygomycosis. Infect Dis Clin North Am 16: 895-914, 2002.

15. Herbrecht R, Denning DW, Patterson TF, et al. Invasive Fungal Infections Group of the European Organisation for Research and Treatment of Cancer and the Global Aspergillus Study Group. Voriconazole versus amphotericin B for primary therapy of invasive aspergillosis. N Engl J Med 347: 408-415, 2002.

16. Walsh TJ, Pappas P, Winston DJ, et al. National Institute of Allergy and Infectious Diseases Mycoses Study Group. Voriconazole compared with liposomal amphotericin B for empirical antifungal therapy in patients with neutropenia and persistent fever. N Engl J Med 346: 225-234, 2002.

17. Walsh TJ, Teppler H, Donowitz GR, et al. Caspofungin versus liposomal amphotericin B for empirical antifungal therapy in patients with persistent fever and neutropenia. N Engl J Med 351: 1391-1402, 2004.

18. Powers JH, Dixon CA, Goldberger MJ. Voriconazole versus liposomal amphotericin B in patients with neutropenia and persistent fever. N Engl J Med 346: 289-290, 2002.

19. Spanakis EK, Aperis G, Mylonakis E. New agents for the treatment of fungal infections: clinical efficacy and gaps in coverage. Clin Infect Dis 43: 1060-1068, 2006.

20. Gubarev N, Separovic J, Gasparovic V, Jelic I. Successful treatment of mucormycosis endocarditis complicated by pulmonary involvement. Thorac Cardiovasc Surg 55: 257-258, 2007.

21. Walsh TJ, Finberg RW, Arndt C, et al. Liposomal amphotericin B for empirical therapy in patients with persistent fever and neutropenia. National Institute of Allergy and Infectious Diseases Mycoses Study Group. N Engl J Med 340: 764-771, 1999.

\footnotetext{
(C) 2008 The Japanese Society of Internal Medicine http://www.naika.or.jp/imindex.html
} 